\title{
Selective THz control of magnetic order: new opportunities from superradiant undulator sources
}

\author{
S Kovalev ${ }^{1}$, Zhe Wang ${ }^{1}$, J-C Deinert ${ }^{1}$, N Awari ${ }^{1,2}$, M Chen ${ }^{1}$, \\ B Green ${ }^{1}$, S Germanskiy ${ }^{1}$, T V A G de Oliveira ${ }^{1,3}$, J S Lee ${ }^{4}$, A Deac ${ }^{1}$, \\ D Turchinovich ${ }^{5,10}$, N Stojanovic ${ }^{6}, \mathbf{S}$ Eisebitt $^{7}$, I Radu $^{7}$, S Bonetti ${ }^{8}$, \\ T Kampfrath ${ }^{9,11}$ and M Gensch ${ }^{1}$ \\ ${ }^{1}$ Helmholtz-Zentrum Dresden-Rossendorf, Bautzner Landstr. 400, 01328 Dresden \\ ${ }^{2}$ University of Groningen, AG 9747 Groningen, Netherlands \\ 3 Technische Universität Dresden, Nöthnitzer Str. 61, 01187 Dresden \\ ${ }^{4}$ Gwangju Institute of Science and Technology (GIST), Gwangju 500-712, Republic of Korea \\ ${ }^{5}$ Fakultät für Physik, Universität Duisburg-Essen, Lotharstr. 1, 47057 Duisburg, Germany \\ ${ }^{6}$ DESY, Notkestr. 85, 22605 Hamburg \\ 7 MBI Max-Born Institute for Nonlinear Optics and Short Pulse Spectroscopy, Max-Born-Str. 2A, \\ 12489 Berlin \\ ${ }^{8}$ Department of Physics, Stockholm University, 10691 Stockholm, Sweden \\ 9 Department of Physics, Freie Universität Berlin, Arnimallee 14, 14195 Berlin \\ ${ }_{10}$ Max Planck Institute for Polymer Research, Ackermannweg 10, 55128 Mainz \\ 11 Fritz Haber Institute of the Max Planck Society, Faradayweg 4-6, 14195 Berlin \\ E-mail: s.kovalev@hzdr.de and m.gensch@hzdr.de
}

\begin{abstract}
Recent advancements of accelerator technology enable the generation of carrier-envelopephase stable $\mathrm{THz}$ pulses with high fields at adjustable high repetition rates. The appropriate choice of $\mathrm{THz}$ radiator allows generation of narrow-band, spectrally dense, multicycle $\mathrm{THz}$ transients of tunable $\mathrm{THz}$ frequency which are ideally suited to selectively excite lowenergy excitations such as magnons or phonons. They also allow one to study the frequency dependence of nonresonant $\mathrm{THz}$-field interactions with various order parameters with high dynamic range. In this paper, we discuss the future prospects of this new type of $\mathrm{THz}$ light source for studying the coherent control of magnetic order based on recent results.
\end{abstract}

Keywords: terahertz, THz control, magnetic order, multicycle pulses

\section{Introduction}

The control of magnetic order by intense transient THz fields is an emerging area of ultra-fast science which has been enabled by the advancements of $\mathrm{THz}$ generation techniques that meanwhile routinely provide $\mathrm{THz}$ fields in the few-10 to $1000 \mathrm{kV} \mathrm{cm}^{-1}$ regime. High-field $\mathrm{THz}$ sources fall into two main classes: table-top sources based on down-conversion of strong near-infrared femtosecond lasers [1] and superradiant
$\mathrm{THz}$ sources based on ultra-short, highly charged electron bunches at modern linear accelerators [2-6].

Various studies have investigated the interaction between $\mathrm{THz}$ fields and magnetic order on sub-THz-cycle timescales and have demonstrated different mechanisms for $\mathrm{THz}$ control. For instance, THz-driven demagnetization by nonresonant ponderomotive acceleration of free or weakly bound electrons via the electric-field component has been observed in conducting samples [7]. Selective and direct interaction of the 
a)

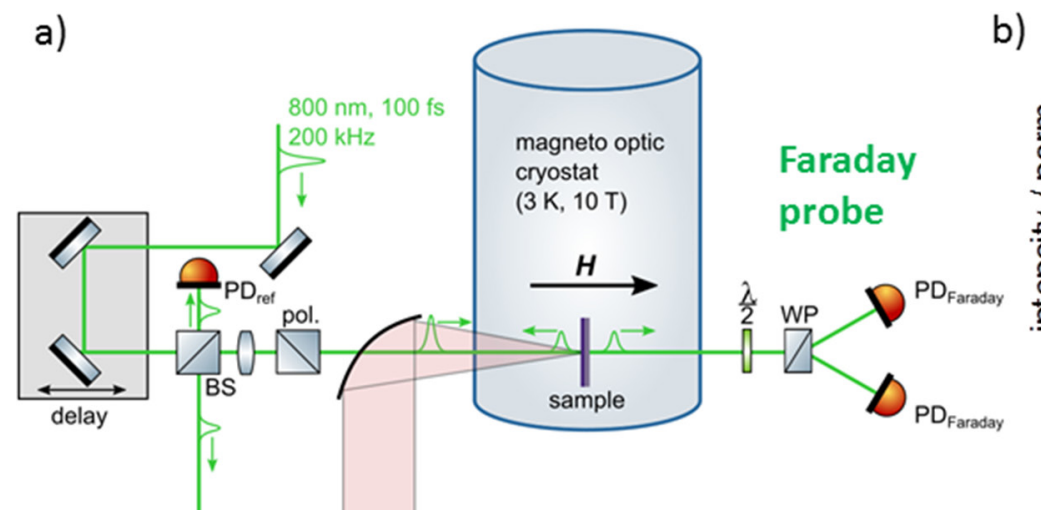

b)

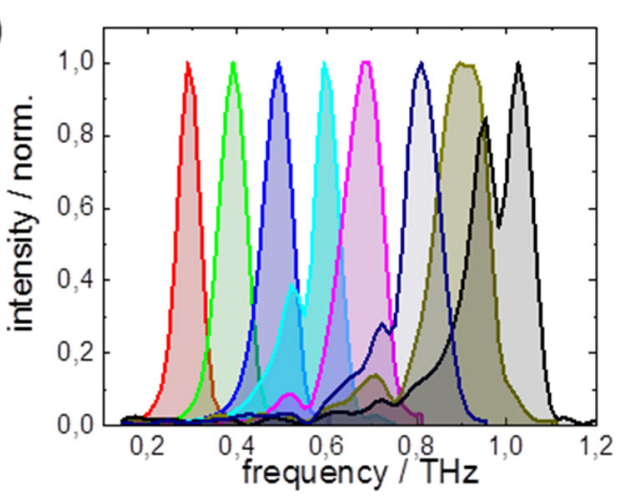

MOKE probe

c)

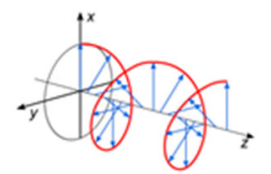

left - circular

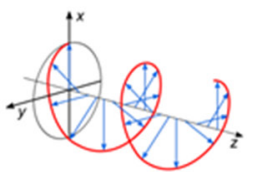

right - circular

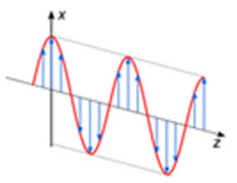

linear

Figure 1. Currently available end station for THz control experiments of magnetic order at TELBE. (a) The measurements can be performed in a commercial $10 \mathrm{~T}$ split-coil-magnet system [20] providing for sample temperatures between 3 and $300 \mathrm{~K}$ and magnetic fields of up to $10 \mathrm{~T}$. In the currently available end station, magnetization dynamics can routinely be probed on ultra-fast timescales with a synchronized femtosecond laser (green line) by transient MOKE and Faraday measurements with a temporal resolution in the few 10 fs regime [14]. Narrow-band, Fourier-limited carrier-envelope-phase (CEP) stable THz pulses are provided as pump pulses at a typical repetition rate of $100 \mathrm{kHz}$ (red line), offering a spectral density that is (already during early stage operation) a factor of 30 higher than those available from laser-based, single-cycle THz pulses [6]. (b) THz spectra of different THz tunes of the TELBE undulator source. During early stage operation frequencies between $0.1-1.2 \mathrm{THz}$ are available with pulse energies of up to $2 \mu \mathrm{J}$. Once operational at its design parameters, $\mathrm{THz}$ fields in the $\mathrm{MV} \mathrm{cm}^{-1}$ regime will become available. (c) Waveplates allow one to control the THz polarization state between circular and linear. This particular picture has been obtained by the author(s) from the Wikimedia website https://commons. wikimedia.org/wiki/File:Circular.Polarization.Circularly.Polarized.Light_Plane.wave_Left.Handed.svg, where it is stated to have been released into the public domain. It is included within this article on that basis.

spin system with the transient magnetic field component via the Zeeman torque has been demonstrated in antiferromagnetic films $[6,8,9]$. More recently, selective THz control of magnetic order via phonon pumping has been successfully exercised $[10,11]$ and the control of atomic-scale magnetic structure has been achieved via resonant excitation of a specific electro-magnon [12]. Most recently low-energy orbital transitions [13] have been utilized to drive large-angle excitations of magnetic lattices.

Many of these experiments benefit strongly from the uniquely high spectral densities of the narrow-band tunable $\mathrm{THz}$ pulses that have become available from superradiant undulator $\mathrm{THz}$ sources at modern linear accelerators. $\mathrm{THz}$ pulses with pulse energies up to the few-100 $\mu \mathrm{J}$ regime and a relative spectral bandwidth between 10 and $20 \%$ can be generated yielding spectral densities that are orders of magnitude beyond that available from laser-based $\mathrm{THz}$ sources $[5,6]$. In this article, we present selected results from the first year of early-stage user operation of the TELBE THz facility [6] that demonstrate the capabilities of this new type of large-scale user facility for studying selective $\mathrm{THz}$ control phenomena.

\section{Methods}

TELBE currently produces multi-cycle THz pulses at a maximum repetition rate of $100 \mathrm{kHz}$, which are tunable between 0.1 and $1.2 \mathrm{THz}$ with pulse energies of up to $2 \mu \mathrm{J}$ while operation at its design parameters will provide pulse energies of up to $100 \mu \mathrm{J}$ in a frequency range up to $3 \mathrm{THz}$ [6] (see figure 1). The generated THz pulses consist of 8 cycles and yield a spectral bandwidth of roughly $20 \%$. The polarization is linear but can be controlled between circular, elliptical and linear by means of appropriate quarter- and half- waveplates. Transient Faraday and MOKE measurements have been established as standard ultra-fast probes for the $\mathrm{THz}$ driven magnetization dynamics. A novel pulse-resolved data acquisition system enables to achieve timing between the probe laser and $\mathrm{THz}$ 
a)

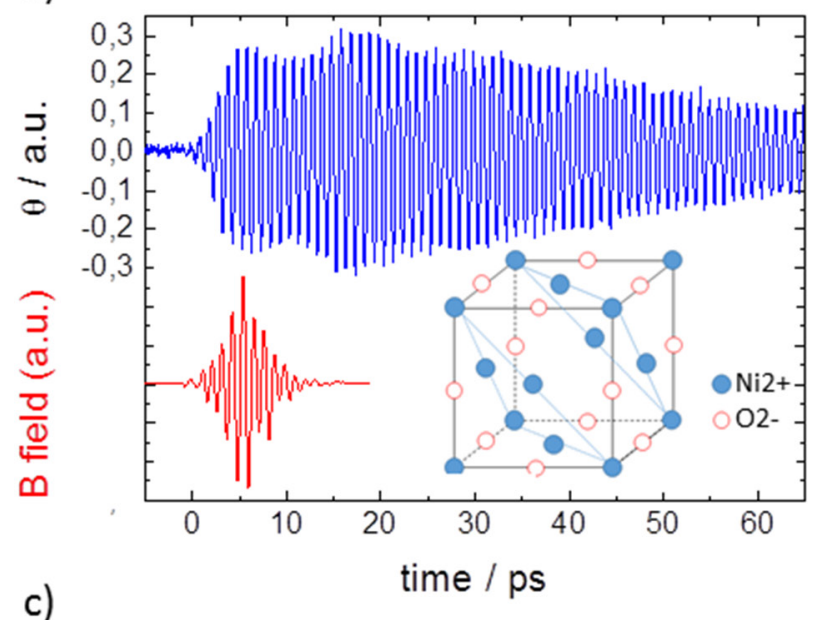

c)

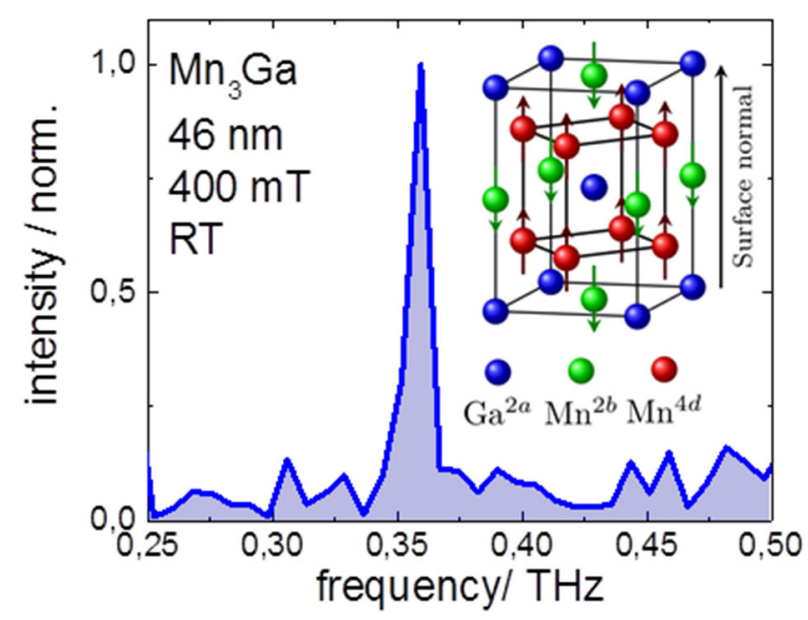

b)
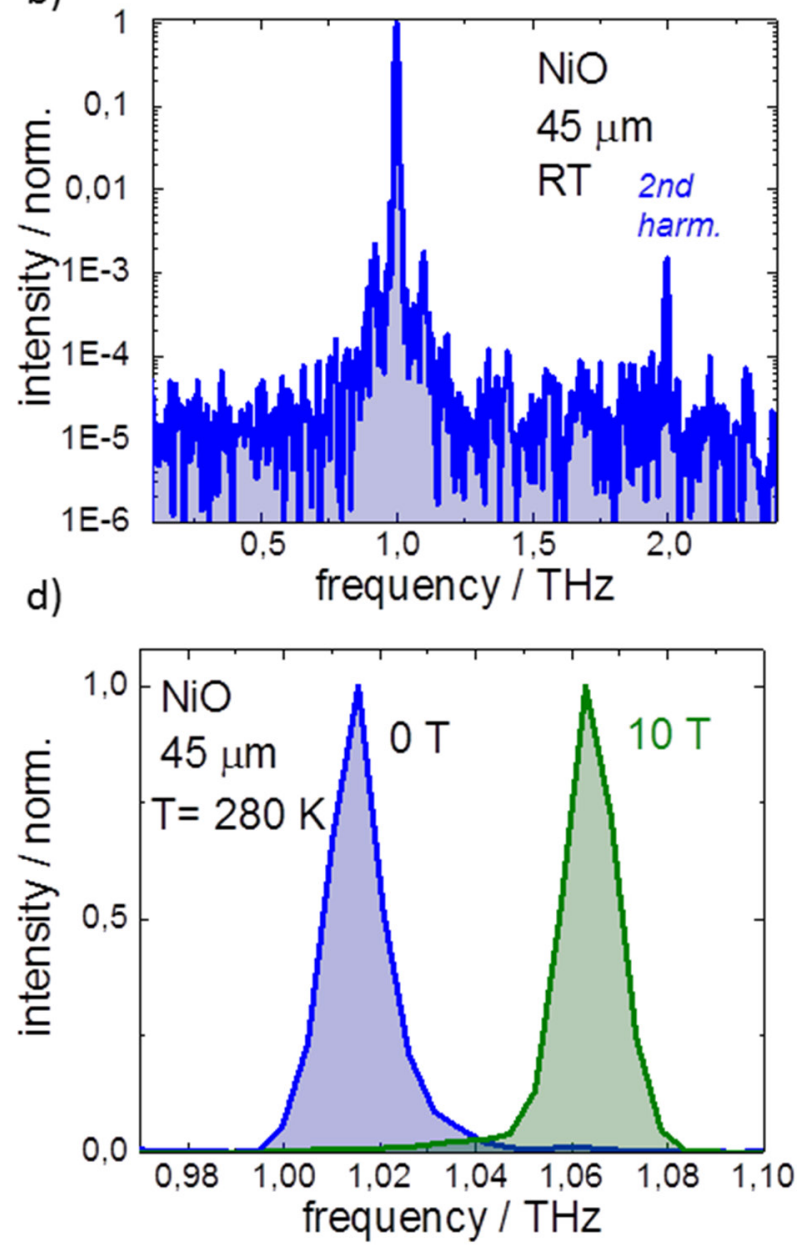

Figure 2. Probing resonant excitation of coherent spin waves by transient Faraday/MOKE spectroscopy (a) THz-driven spinwave experiment on a $45 \mu \mathrm{m} \mathrm{NiO}$ film, where the dynamics is probed by transient Faraday measurements with a $800 \mathrm{~nm}$ fs laser. The frequency of the pulsed $\mathrm{THz}$ magnetic field (red) can be tuned into resonance with the antiferromagnetic (AFM) mode of $\mathrm{NiO}$ at $1 \mathrm{THz}$ yielding a spectral density that is by a factor of 30 higher than those available from laser-based single cycle THz pulses [6]. (b) Pulse-resolved detection in combination with the high spectral density and the high repetition rate of $100 \mathrm{kHz}$ allows to achieve a dynamic range of better than $10^{4}$ in routine measurements [14] which permits observation of the recently described emergence of higher-order features in the Faraday signal [9]. (c) The excellent dynamic range is important for the transfer of the concept of THz-driven spinwave excitations probed by time-resolved Faraday/MOKE measurements to thin films. As an example, the recent Faraday measurement of the coherently driven ferromagnetic (FM) resonance in a $46 \mathrm{~nm}$ thin $\mathrm{Mn}_{3} \mathrm{Ga}$ film is shown [18]. (d) The high dynamic range also aids experiments in extreme sample environments such as high magnetic fields and low temperatures. Shown here is an example of the effect of a $10 \mathrm{~T}$ magnetic field on the AFM resonance in $\mathrm{NiO}$ which is shifted to higher $\mathrm{THz}$ frequencies by $\sim 0.06 \mathrm{THz}[16]$.

pulses with a resolution of $12 \mathrm{fs}$ (rms) and an exceptional dynamic range exceeding $10^{6}$ in experiments that probe $\mathrm{THz}$ driven dynamics in solids [14].

\section{Results}

Figure 2 shows experimental results of one typical class of early-stage experiments at TELBE. A multicycle $\mathrm{THz}$ pulse is tuned into resonance with specific magnetic modes and the coherent excitation is traced on sub-THz-cycle timescales by the transient Faraday rotation of a synchronized NIR fs laser. The first benchmark experiment, results of which are shown in figures 2(a) and (b), has been performed on the prototypical antiferromagnet $\mathrm{NiO}$ [15].

The antiferromagnetic resonance in this material at a frequency of $\sim 1 \mathrm{THz}$ frequency has been utilized in the seminal first demonstration experiments of $\mathrm{THz}$ coherent control of spinwaves using a laser-based $\mathrm{THz}$ radiation source [8]. Using the spectrally dense narrow-band TELBE THz pulses allows one to achieve a significantly larger spin deflection amplitude at similar pulse energies, since the energy is more efficiently directed selectively into the narrow resonance [6]. A dynamic range of better than $10^{4}$ can be achieved easily on short measurement timescales. This permits the observation of only recently demonstrated higher-order effects such as the occurrence of a 2nd harmonic component of the $1 \mathrm{THz}$ fundamental frequency in the Faraday response as demonstrated recently [9] with a superior signal-to-noise ratio. The increased sensitivity furthermore enables detection of such $\mathrm{THz}$-driven spin excitations even in materials with small Verdet constants [17] or in ultra-thin films. An example is shown in figure 2(c), where the THz-driven ferromagnetic (FM) mode in an only $46 \mathrm{~nm}$ thick ferrimagnetic pseudo-Heusler-alloy $\mathrm{Mn}_{3} \mathrm{Ga}$ film 
a)

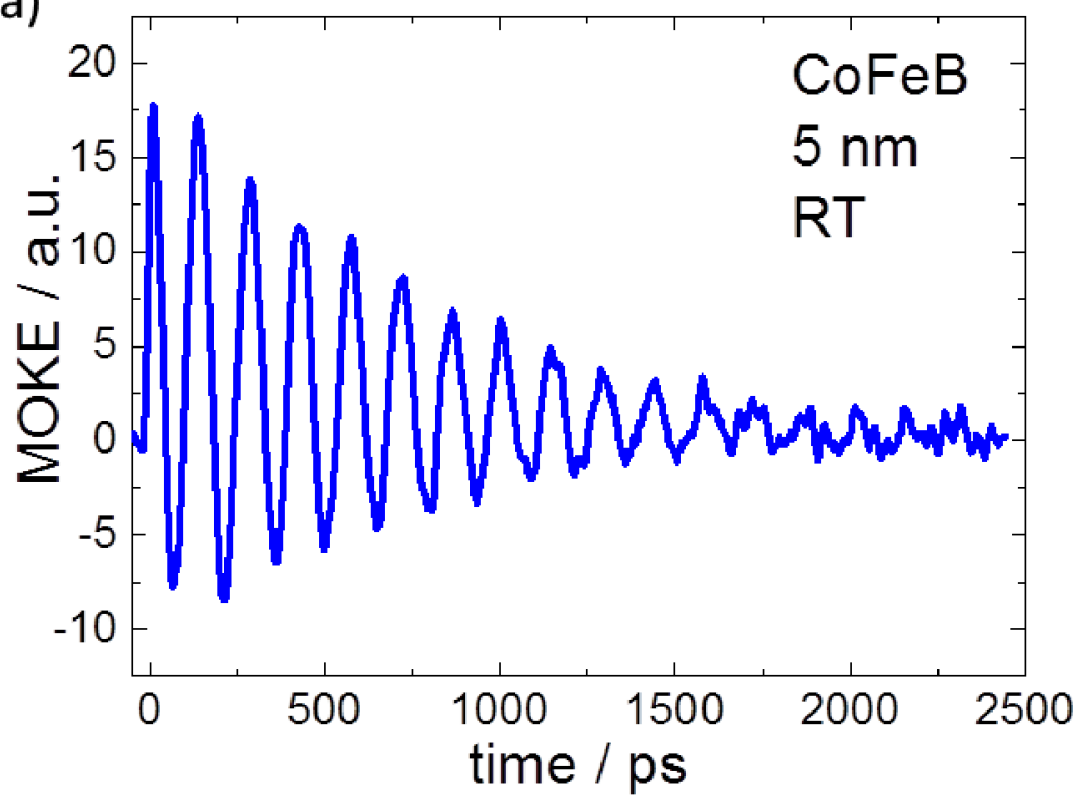

b)

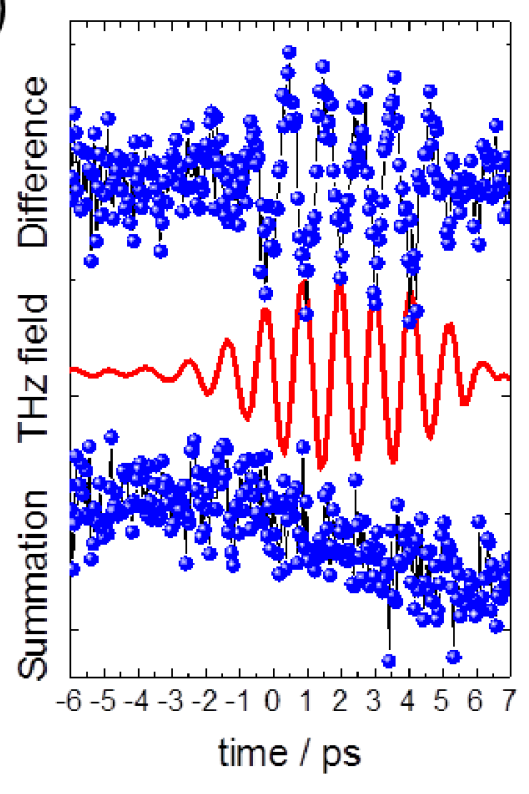

Figure 3. Probing non-resonant THz driven demagnetization in ferromagnetic films by transient MOKE measurements. (a) Multicycle $\mathrm{THz}$ pulses drive the recently observed demagnetization in a $5 \mathrm{~nm}$ CoFeB film. The initiated demagnetization leads to the excitation of the ferromagnetic resonance on nanosecond timescales which can be followed at TELBE by shifting the electronic phase between the probe laser and the accelerator masterclock. (b) The onset of the demagnetization on ultra-fast timescales can be probed at few 10 fs timescales by measuring transient MOKE response at THz fields of opposite polarity (prepared by use of THz HWPs). Plotting the sum and difference of the derived response allows one to disentangle the fast coherent response following the driving THz field (Difference) and the picosecondtimescale noncoherent demagnetization response (Sum). The THz excitation pulse is also shown (red).

has been observed at a resonance frequency of $0.359 \mathrm{THz}$. In this experiment another advantage of selective $\mathrm{THz}$ excitation becomes apparent: When comparing with concurrent laser-stimulated $\mathrm{THz}$ emission measurements, one finds that the observed resonance frequency is shifted noticeably by $0.09 \mathrm{THz}$ to lower frequencies in comparison to the TELBE experiment [18]. Subsequent temperature dependence measurements of the $\mathrm{THz}$ emission process [19] show that this can be understood by an increase of the average sample temperature by $15 \mathrm{~K}$ due to the off-resonant laser excitation at $800 \mathrm{~nm}$ corresponding to $375 \mathrm{THz}$ or $1.55 \mathrm{eV}$ which is 3 orders of magnitude higher than the energy of the actual resonance. This type of residual excitation of the multiple energy degrees of freedom between the $\mathrm{eV}$ and $\mathrm{meV}$ range at once can be completely avoided when narrow-band $\mathrm{THz}$ pulses are used to exclusively address the FM resonance in $\mathrm{Mn}_{3} \mathrm{Ga}$ at $0.36 \mathrm{THz}(1.49 \mathrm{meV})$ as has been done in the TELBE experiment [18].

The end station at the TELBE facility has recently been equipped with a split-coil cryomagnet [20] that allows one to employ external magnetic fields of up to $10 \mathrm{~T}$ at temperatures between 3 and $300 \mathrm{~K}$. Again utilizing $\mathrm{NiO}$ as a benchmark material, this enabled the observation of a clear shift of the AFM mode towards higher frequencies by $0.06 \mathrm{THz}$ when applying a magnetic field of $10 \mathrm{~T}$ in the Faraday configuration (i.e. H\|lk) [16]. To this end, the near-Gaussian-beamlike spatial properties of undulator radiation proves of high importance in keeping the inevitable transport losses of $\mathrm{THz}$ radiation into the $10 \mathrm{~T}$ split-coil magnet at minimum and by allowing achievement of close-to-optimal focal spot sizes on the sample. In combination with the high repetition rate this enables a dynamic range of better than $10^{3}$. The decrease in dynamic range compared to ambient conditions is because of the loss in fluency/THz field due to the larger focal length enforced by the $\sim 200 \mathrm{~mm}$ distance between the sample and the optical viewports of the magnet.

Another class of experiments that benefits from the narrow bandwidth and tunability are those investigating the frequency dependence of the efficiency of THz-driven phenomena. An important consequence of the interaction of strong $\mathrm{THz}$ transients with matter is the acceleration of free or weakly bound electrons by the transient $\mathrm{THz}$ electric field component. Here, the interaction mechanism is based on the ponderomotive force exerted by the THz field and is thus scaling up with $1 / \nu^{2}$, where $\nu$ is the radiation frequency. This means that processes based on this interaction, e.g. the $\mathrm{THz}$ driven demagnetization of metallic ferromagnetic thin films [7], should become significantly more efficient at lower THz frequencies. TELBE has meanwhile successfully been utilized to drive demagnetization in $\mathrm{CoFeB}$ thin films at different $\mathrm{THz}$ frequencies [21]. An example for excitation with a $1 \mathrm{THz}$ pulse is shown in figure 3. The induced ultrafast demagnetization also leads to the subsequent coherent excitation of the FM resonance at a few $\mathrm{GHz}$ frequency which can be followed over ns time scales by electronically shifting the phase between the probe laser and the electron accelerator (shown in figure 3(a)). On ultrafast timescales the $\mathrm{THz}$ polarization control by half-waveplates allows one to perform measurements of the transient MOKE signal at exactly antiparallel THz magnetic field directions. This enables the detection of the actual demagnetization (quadratic with respect to the $\mathrm{THz}$ field) from the resulting summation signal (see figure 3(b)) [21], and to disentangle it 
a)

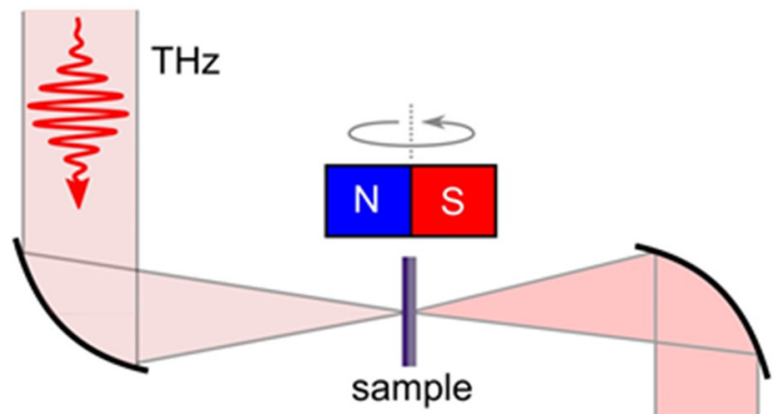

b)

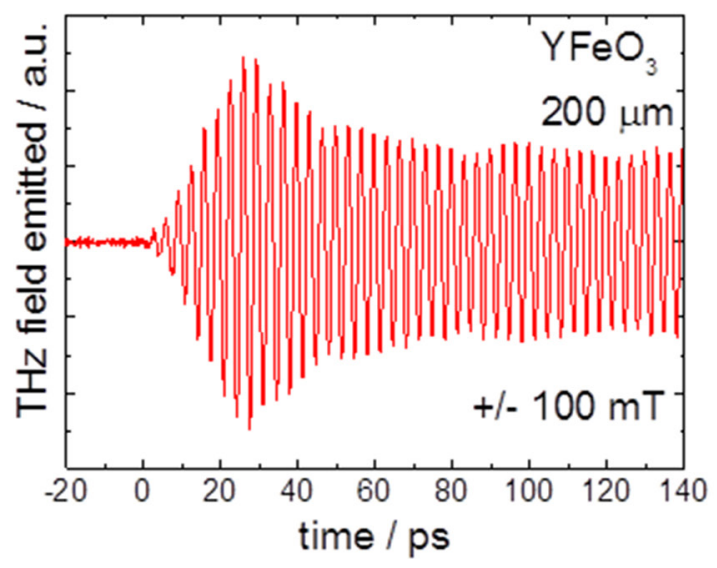

c)

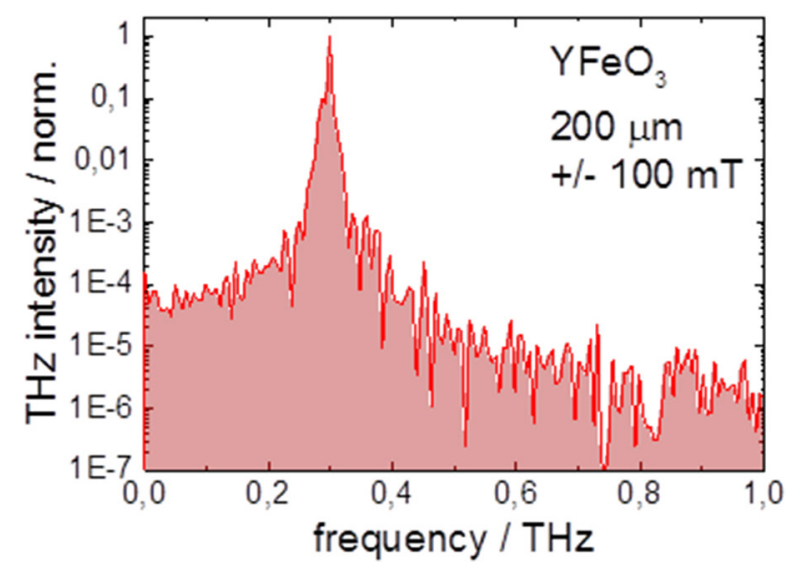

Figure 4. Selective driving and probing of a spinwave by THz emission spectroscopy: (a) Experimental set-up for THz emission spectroscopy. The incident narrow-band spectrally dense THz pulses drive spin excitations coherently. An electromagnetic wave is reemitted, with a field strength that is directly proportional to the 2 nd derivative of the temporal magnetization change. The re-emitted field can be separated from the excitation pulse by subtraction of measurements for two opposite external magnetic fields. (b) Emitted THz transient of a resonantly driven $\mathrm{FM}$ spin excitation in $\mathrm{YFeO}_{3}$ and (c) corresponding intensity spectrum. A dynamic range of better than $10^{6}$ has been achieved.

from the coherent precession (linear with the THz field) that can be isolated by taking the difference signal.

\section{Discussion and conclusion}

This paper demonstrates the potential of superradiant undulator facilities for research on magnetic materials that requires selective excitation with intense CEP stable, narrow-band $\mathrm{THz}$ light. Transient Faraday/MOKE spectroscopy has become the standard technique to probe selective THz control of magnetic order in the early-stage user experiments at TELBE. The reason for this is its comparatively straightforward implementation. The technique benefits from the high spectral density and the high repetition rate by achieving very high dynamic range. Thereby, THz-driven dynamics can be investigated in systems and in sample environments that are presently inaccessible by all-laser-based approaches. Another easy-to-implement technique that benefits tremendously from the combination of high field and high repetition rate is $\mathrm{THz}$ emission spectroscopy (see figure 4).

Over the past 12 years several seminal experiments demonstrated that rapid changes of the magnetic state [22, 23], ultrafast spin currents [24] and, more recently, spinwave excitations $[18,25,26]$ can lead to the emission of transient $\mathrm{THz}$ pulses. These pulses carry information on the spin dynamics involved (e.g. timescales or the nature of the magnetic resonances involved). Figures 4(b) and (c) shows a first example experiment of this type at TELBE where $\mathrm{THz}$ emission has been observed after selective excitation of the ferromagnetic mode at $0.3 \mathrm{THz}$ in the model antiferromagnet $\mathrm{YFeO}_{3}$ [25]. In this benchmark experiment, an excellent dynamic range of better than $10^{5}$ has been achieved. The ultimate advantage of this technique is that the only perturbation of the sample is the selective excitation of the specific low energy resonance of interest with the narrowband $\mathrm{THz}$ excitation pulse. Parasitic 

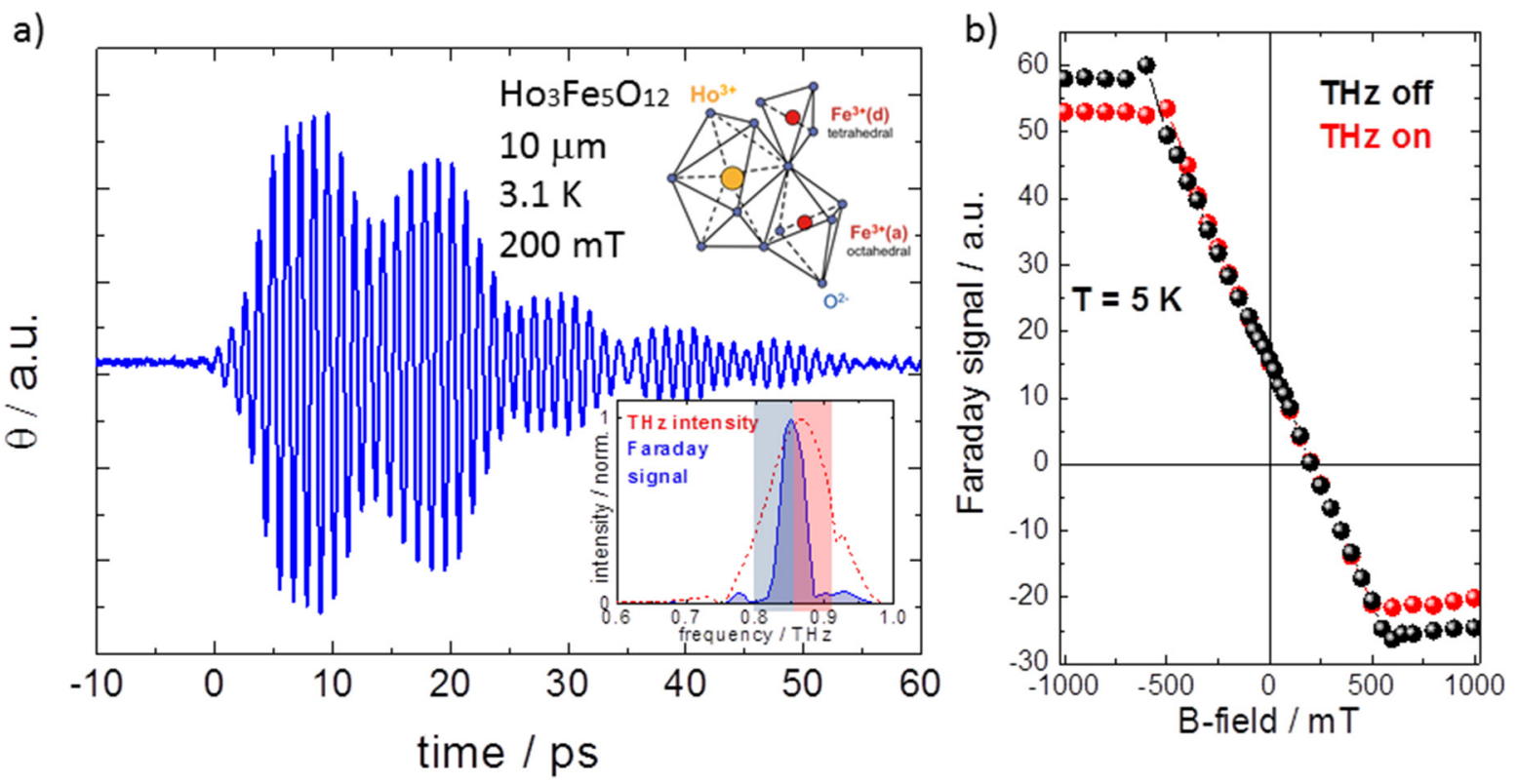

Figure 5. Effect of the average heating via direct THz lattice pumping in HoIG. (a) Transient Faraday signal after resonant THz excitation of $10 \mu \mathrm{m}$ thick $\mathrm{Ho}_{3} \mathrm{Fe}_{5} \mathrm{O}_{12}$ (HoIG) sample. (inset) Spectrum of the THz excitation pulse (red) tuned in resonance with the optical magnon mode of HoIG (blue shaded area)..The THz spectrum also overlaps with the $\mathrm{Ho}^{3+}$ crystal field resonance between 0.85 and $0.89 \mathrm{THz}$ (red shaded). (b) A clearly observable temperature increase is quantified to be less than $15 \mathrm{~K}$ by measurement of the hysteresis with and without $\mathrm{THz}$ radiation impacting on the sample. The average $\mathrm{THz}$ power incident on the sample in this experiment was roughly $100 \mathrm{~mW}$.

effects from additional probe light pulses are completely avoided. The dynamics (e.g. dephasing) of the coherently excited magnetic mode is followed by determination of the properties of the re-emitted $\mathrm{THz}$ wave.

Other versatile probe techniques for studying THz-driven changes of magnetic order that require more efforts in implementation are time-resolved scanning nearfield optical microscopy (TR-SNOM) $[27,28]$ and time, angle and spinresolved photoelectron spectroscopy (TR-ARPES) [29]. These two techniques fully rely on the high repetition rate presently uniquely available from TELBE and shall enable probing of $\mathrm{THz}$ driven dynamics on nanometer length scales and to follow the $\mathrm{THz}$ field induced changes directly in the electronic structure. End stations for both of these techniques are currently under development $[30,31]$.

Besides the advantages of the combination of high-field and high-repetition-rate $\mathrm{THz}$ sources one obvious obstacle arises when a significant part of the incident $\mathrm{THz}$ power leads to the subsequent heating of the sample. In the current early stage operation TELBE provides average powers of $100 \mathrm{~mW}$ in the frequency range of $0.1-1.2 \mathrm{THz}$, but these powers will reach up to $10 \mathrm{~W}$ once the design parameters are achieved [6]. So far, samples that exhibited exclusively spin resonances in the addressed $\mathrm{THz}$ frequency range did not show a detectable increase of the average temperature, although the indication of magnon-lattice coupling was observed in the decay dynamics of THz-excited magnon in some experiments [26]. This has been different in cases where the spectral content of the $\mathrm{THz}$ excitation could directly couple to lattice degrees of freedom such as phonon modes [32]. In a recent study on THz-driven spin dynamics in holmium iron garnet (HoIG), the $\mathrm{THz}$ excitation pulse not only overlapped with a magnon but also with a crystal-field resonance (see figure 5) [33]. In this case, a clear magnitude change of the hysteresis has been observed that could be associated with a temperature increase of $\sim 15 \mathrm{~K}$ (see figure $5(\mathrm{~b})$ ), which appeared due to the low thermal conductivity of HoIG at the steady-state temperature of $5 \mathrm{~K}$.

The motivation for the experiments shown in this paper stems mostly from an interest in: (i) background free spectroscopic measurements in the electronic ground state at high dynamic range (e.g. magnetic field and temperature dependence of resonances in $\mathrm{NiO}$ and $\mathrm{YFeO}_{3}$ or the dependence of the FM resonance on the composition of $\mathrm{Mn}_{3-x} \mathrm{Ga}$ nanofilms) or (ii) a better understanding of the underlying physical processes of recently discovered $\mathrm{THz}$ control phenomena like the demagnetization in $\mathrm{CoFeB}$ films [7] by determination of their frequency dependence. Furthermore the dephasing of the coherent excitations can be followed and analyzed in the timedomain which allows to directly observe how and when the $\mathrm{THz}$ energy initially pumped selectively into a specific low energy mode such as an optical magnon is distributed to other degrees of freedom in the solid. Once TELBE can be operated with its design bunch charge of $1 \mathrm{nC}$, the pulse energies are expected to increase by 2 orders of magnitude (see figure 6) corresponding to field strengths $>1 \mathrm{MV} \mathrm{cm}^{-1}$ or few 100 $\mathrm{mT}$. In this case, experiments will be able to explore selective $\mathrm{THz}$ control of magnetic degrees of freedom beyond the currently available perturbative regime and access intriguing phenomena such as $\mathrm{THz}$ induced spin flip processes. Directing such exceptionally high fields selectively into narrow magnetic resonances will help to avoid recently discussed destructive effects of the $\mathrm{THz}$ electric fields [34] since off-resonant spectral components are kept at a minimum. 


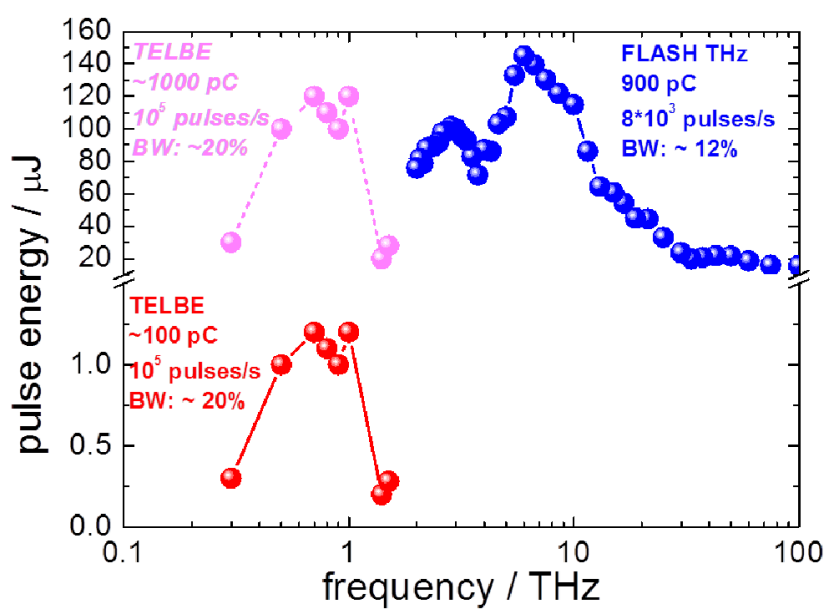

Figure 6. Pulse energies available between 0.1 and $100 \mathrm{THz}$ from the two presently existing superradiant undulator based user facilities. The pulse energies scale quadratically with the bunch charge. Provided the electron bunch can be made short enough, this yields pulse energies in the $100 \mu \mathrm{J}$ regime for bunch charges in the $1 \mathrm{nC}$ regime as already available with repetition rates up to 8000 pulses s $^{-1}$ from the FLASH THz source [5] (blue). TELBE can currently be operated at a high repetition rate of $100 \mathrm{kHz}$ but only with a moderate bunch charge of below $100 \mathrm{pC}$ yielding pulse energies in the $1 \mu \mathrm{J}$ regime (red). After the planned upgrade, bunch charges of up to $1 \mathrm{nC}$ should be available yielding pulse energies of up to $100 \mu \mathrm{J}$ in the frequency range between 0.2 and $1.2 \mathrm{THz}$ [6].

\section{Acknowledgment}

NA, AD and MG acknowledge support from the European Commission's Horizon 2020 research and innovation programme, under Grant Agreement No DLV 737038 (TRANSPIRE). BG, SK and MG acknowledge support from the European Cluster of Advanced Laser Light Sources (EUCALL) project, which has received funding from the European Union's Horizon 2020 research and innovation programme under Grant Agreement No 654220. TVAGO and MG acknowledges support from the German Federal Ministry of Education and Research (BMBF) for the project grant No 05K16ODC (TiNa). JSL acknowledges support from Basic Science Research Program through the National Research Foundation of Korea (NRF), funded by the Ministry of Science, ICT and Future Planning (MSIP) (2015R1A5A1009962, 2015R1A1A1A05001560). DT acknowledges the financial support from the European Commission (EU Career Integration Grant EU CIG 334324 LIGHTER) and from the Max Planck Society. SB acknowledges support from the European Research Council, Starting Grant 715452 'MAGNETIC-SPEED- LIMIT'. NA acknowledges support through the Ubbo Emmius Program of the University of Groningen. TK acknowledges funding through the ERC H2020 CoG project TERAMAG/Grant No. 681917. NS acknowledges support from Deutscher Akademischer Austauschdienst (DAAD) grant No. 57219839 and Bundesministerium fuer Bildung und Forschung BMBF grant No. 05K12CH4). SE and IR acknowledge funding from BMBF through project $05 \mathrm{~K} 16 \mathrm{BCA}$ Femto-THz-X.

We thank the ELBE team for operating the TELBE THz sources during the experiments.
Certain images in this publication have been obtained by the author(s) from the Wikipedia/Wikimedia website, where they were made available under a Creative Commons licence or stated to be in the public domain. Please see individual figure captions in this publication for details. To the extent that the law allows, IOP Publishing [and full partner name (if applicable)] disclaim any liability that any person may suffer as a result of accessing, using or forwarding the image(s). Any reuse rights should be checked and permission should be sought if necessary from Wikipedia/Wikimedia and/or the copyright owner (as appropriate) before using or forwarding the image(s).

\section{ORCID iDs}

J-C Deinert (1) https://orcid.org/0000-0001-6211-0158

B Green (1) https://orcid.org/0000-0002-7184-5620

S Bonetti (1) https://orcid.org/0000-0001-9352-2411

M Gensch (1) https://orcid.org/0000-0001-7755-1618

\section{References}

[1] Kampfrath T et al 2013 Resonant and nonresonant control over matter and light by intense terahertz transients Nat. Photon. 7680

[2] Gensch M et al 2008 New infrared undulator beamline at FLASH Infrared Phys. Technol. 5423

[3] Hoffmann M C et al 2011 Coherent single-cycle pulses with $\mathrm{MV} / \mathrm{cm}$ field strengths from a relativistic transition radiation light source Opt. Lett. 364473

[4] Wu Z et al 2013 Intense terahertz pulses from SLAC electron beams using coherent transition radiation Rev. Sci. Instrum. 84022701

[5] Stojanovic N et al 2013 Accelerator- and laser-based sources of high-field terahertz pulses J. Phys. B: At. Mol. Phys. 46192001

[6] Green B et al 2016 High-field high-repetition-rate sources for the coherent THz control of matter Sci. Rep. 622256

[7] Bonetti S et al $2016 \mathrm{THz}$-driven ultrafast spin-lattice scattering in amorphous metallic ferromagnets Phys. Rev. Lett. 117087205

[8] Kampfrath T et al 2011 Coherent terahertz control of antiferromagnetic spin waves Nat. Photon. 531

[9] Baierl S et al 2016 Terahertz-driven nonlinear spin response of antiferromagnetic nickel oxide Phys. Rev. Lett. 117107201

[10] Nova T F et al 2017 An effective magnetic field from optically driven phonons Nat. Phys. 13132

[11] Maehrlein S et al Revealing spin-phonon interaction in ferrimagnetic insulators by ultrafast lattice excitation (arXiv:1710.02700)

[12] Kubacka T et al 2017 Large-amplitude spin dynamics driven by a THz pulse in resonance with an electromagnon Science 3436177

[13] Baierl S et al 2016 Nonlinear spin control by terahertz-driven anisotropy fields Nat. Photon. 10715

[14] Kovalev S et al 2017 Probing ultra-fast processes with high dynamic range at 4th-generation light sources: arrival time and intensity binning at unprecedented repetition rates Struct. Dyn. 4024301

[15] Sänger I et al 2006 Distribution of antiferromagnetic spin and twin domains in NiO Phys.Rev. B 74144401

[16] Wang $\mathrm{Z}$ et al in preparation 
[17] Hecht E 2009 Optik (München/Wien: Oldenbourg Verlag)

[18] Awari N et al 2016 Narrow-band tunable terahertz emission from ferrimagnetic $\mathrm{Mn}_{3-x} \mathrm{Ga}$ thin films Appl. Phys. Lett. 109032403

[19] Awari N et al in preparation

[20] www.oxford-instruments.com/products/ cryogenic-environments/superconductingmagnet-systems/cryogen-free-magnets/ cryogen-free-magneto-optical-superconducting-old

[21] Bonetti $\mathrm{S}$ et al in preparation

[22] Beaurepaire E, Turner G M, Harrel S M, Beard M C, Bigot J-Y and Schmuttenmaer C A 2004 Coherent terahertz emission from ferromagnetic films excited by femtosecond laser pulses Appl. Phys. Lett. 843465

[23] Hilton D J, Averitt R D, Meserole C A, Fisher G L, Funk D J, Thompson J D and Tayl A Jor 2004 Terahertz emission via ultrashort-pulse excitation of magnetic metal films $O p t$. Lett. 291805

[24] Kampfrath T, Battiato M, Maldonado P, Eilers G, Noetzold J, Maehrlein S, Zbarsky V, Freimuth F, Mokrousov Y and Bluegel S 2013 Terahertz spin current pulses controlled by magnetic heterostructures Nat. Nanotechnol. 8256
[25] Kim T H et al 2014 Coherently controlled spin precession in canted antiferromagnetic $\mathrm{YFeO}_{3}$ using terahertz magnetic field Appl. Phys. Express 7093007

[26] Jin Z, Mics Z, Ma G, Cheng Z, Bonn M and Turchinovich D 2013 Single pulse terahertz coherent control of spin resonance in the canted antiferro-magnet $\mathrm{YFeO}_{3}$, mediated by dielectric anisotropy Phys. Rev. B 87094422

[27] Kehr S et al 2017 FEL-based near-field infrared to THz nanoscopy Synchrotron Radiat. News 3031

[28] Sternbach A J et al 2017 Artifact free time resolved near-field spectroscopy Opt. Express 2528589

[29] Gedik N et al 2017 Photoemission of quantum materials Nat. Phys. 131029

[30] Kuschewski F et al 2015 Optical nanoscopy of transient states in condensed matter Sci. Rep. 512582

[31] www.unidue.de/imperia/md/content/usd10/usd_10_book_of_ abstracts_rev1.pdf

[32] Miller T A et al 2015 Terahertz field control of in-plane orbital order in $\mathrm{La}_{0.5} \mathrm{Sr}_{1.5} \mathrm{MnO}_{4}$ Nat. Commun. 68175

[33] Radu I et al in preparation

[34] Shalaby M et al The terahertz frontier of ultrafast coherent magnetic switching: terahertz-induced demagnetization of ferromagnets (arXiv:1506.05397) 